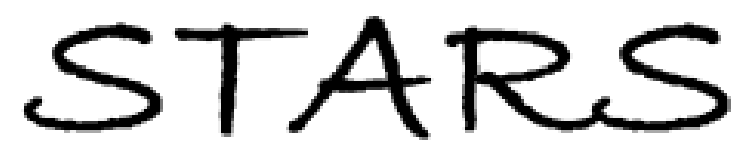

University of Central Florida

STARS

Faculty Bibliography 2010s

Faculty Bibliography

$1-1-2010$

\title{
Surface and grain-boundary scattering in nanometric Cu films
}

Tik Sun

University of Central Florida

Bo Yao

University of Central Florida

Andrew P. Warren

University of Central Florida

Katayun Barmak

Michael F. Toney

See next page for additional authors

Find similar works at: https://stars.library.ucf.edu/facultybib2010

University of Central Florida Libraries http://library.ucf.edu

This Article is brought to you for free and open access by the Faculty Bibliography at STARS. It has been accepted for inclusion in Faculty Bibliography 2010 s by an authorized administrator of STARS. For more information, please contactSTARS@ucf.edu.

\section{Recommended Citation}

Sun, Tik; Yao, Bo; Warren, Andrew P.; Barmak, Katayun; Toney, Michael F.; Peale, Robert E.; and Coffey, Kevin R., "Surface and grain-boundary scattering in nanometric Cu films" (2010). Faculty Bibliography 2010s. 848.

https://stars.library.ucf.edu/facultybib2010/848

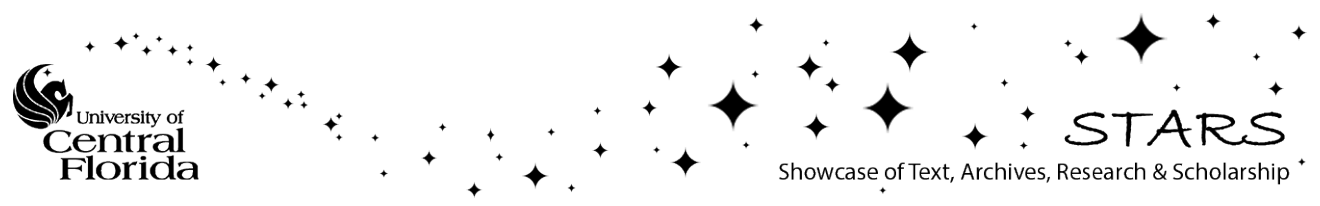


Authors

Tik Sun, Bo Yao, Andrew P. Warren, Katayun Barmak, Michael F. Toney, Robert E. Peale, and Kevin R. Coffey 


\title{
Surface and grain-boundary scattering in nanometric $\mathrm{Cu}$ films
}

\author{
Tik Sun, ${ }^{1}$ Bo Yao, ${ }^{1}$ Andrew P. Warren, ${ }^{1}$ Katayun Barmak, ${ }^{2}$ Michael F. Toney, ${ }^{3}$ Robert E. Peale, ${ }^{4}$ and Kevin R. Coffey 1,4 \\ ${ }^{1}$ Advanced Materials Processing and Analysis Center, University of Central Florida, \\ 4000 Central Florida Boulevard, Orlando, Florida 32816, USA \\ ${ }^{2}$ Materials Research Science and Engineering Center and Department of Materials Science and Engineering, \\ Carnegie Mellon University, 5000 Forbes Avenue, Pittsburgh, Pennsylvania 15213, USA \\ ${ }^{3}$ Stanford Synchrotron Radiation Lightsource, 2575 Sand Hill Road, Menlo Park, California 94025, USA \\ ${ }^{4}$ Department of Physics, University of Central Florida, 4000 Central Florida Boulevard, Orlando, Florida 32816, USA
}

(Received 10 September 2009; revised manuscript received 9 March 2010; published 28 April 2010)

\begin{abstract}
We report a quantitative analysis of both surface and grain-boundary scattering in $\mathrm{Cu}$ thin films with independent variation in film thickness ( 27 to $158 \mathrm{~nm}$ ) and grain size (35 to $425 \mathrm{~nm}$ ) in samples prepared by subambient temperature film deposition followed by annealing. Film resistivities of carefully characterized samples were measured at both room temperature and at $4.2 \mathrm{~K}$ and were compared with physical models that include the effects of surface and grain-boundary scattering. Grain-boundary scattering is found to provide the strongest contribution to the resistivity increase. However, a weaker, but significant, role is observed for surface scattering. We find that the data are best fit when the Mayadas and Shatzkes' model of grain-boundary scattering and the Fuchs and Sondheimer's model of surface scattering resistivity contributions are combined using Matthiessen's rule (simple addition of resistivities). This finding implies that grain-boundary scattering preserves the component of electron momentum parallel to the grain-boundary plane. Using Matthiessen's rule, we find our data are well described by a grain-boundary reflection coefficient of 0.43 and a surface specularity coefficient of 0.52 . This analysis finds a significantly lower contribution from surface scattering than has been reported in previous works and we attribute this difference to the careful quantitative microstructural characterization performed on our samples. The effects of surface roughness, impurities, voids, and interactions between surface and grain-boundary scattering are also examined and their importance is evaluated.
\end{abstract}

DOI: 10.1103/PhysRevB.81.155454

PACS number(s): 73.63.-b, 73.23.-b, 73.43.Fj, 72.10.Fk

\section{INTRODUCTION}

The classical resistivity size effect, wherein conductors with dimensions on the order of the mean free path of electrons (39 $\mathrm{nm}$ for $\mathrm{Cu}$ at room temperature) exhibit higher resistivity than bulk conductors, was noted by Thompson ${ }^{1}$ in 1901. There are a number of scattering mechanisms that can lead to this resistivity increase. The two mechanisms of greatest scientific and technological interest are surface scattering (evidenced by the thickness dependence of the resistivity increase) and grain-boundary scattering (evidenced by the grain size dependence of the resistivity increase). To quantify the relative contributions of grain-boundary and surface scattering to the classical size effect, the following experimental conditions must be met. ${ }^{2}$

(i) The scattering interfaces (for films, the top and bottom surfaces) of the conductor must be identical.

(ii) The sample set must include independent variation in the conductor's average grain size and of the spacing between exterior surfaces (thickness for films).

(iv) The structure (e.g., continuity/voiding, thickness, roughness, grain size) of the conductor must be characterized in detail. For grain size, statistically significant populations (typically $10^{3}$ grains per sample) must be measured.

A detailed review of the literature on the size effect over the past 100 years (combining the review of the first 80 years 1901-1983, by Sambles ${ }^{2}$ and our own review from 19832003, when we undertook our studies in $\mathrm{Cu}$ films ${ }^{3}$ ) showed that no prior experimental study had satisfied these three essential requirements. As a result, there has been much con- fusion regarding the relative contributions of surface scattering and grain-boundary scattering to the classical resistivity size effect. In this work, we present a definitive quantification of these two contributions to the classical size effect for the technologically important example of copper.

In previous work, ${ }^{3,4}$ we investigated separately the room temperature resistivity of $\mathrm{SiO}_{2} / \mathrm{Cu} / \mathrm{SiO}_{2}$ samples and the liquid $\mathrm{He}$ temperature resistivities of $\mathrm{SiO}_{2} / \mathrm{Cu} / \mathrm{SiO}_{2}$ and $\mathrm{SiO}_{2} / \mathrm{Ta} / \mathrm{Cu} / \mathrm{Ta} / \mathrm{SiO}_{2}$ samples. The grain size and thickness of the $\mathrm{Cu}$ layer were both varied, and the resistivity data were found to be consistent with grain-boundary scattering as the dominant mechanism. The presence of a surface scattering contribution to the size effect could not be conclusively demonstrated. In this work, we combine the room temperature $(296 \mathrm{~K}$, the typical temperature in the laboratory) and low-temperature $(4.2 \mathrm{~K})$ resistivity data for both sample types. The combined data allows us to confirm the presence of a weak surface scattering contribution to the resistivity size effect. Unexpectedly, we find that there is no apparent interaction between the surface and grain-boundary scattering (i.e., they add independently). We also examine surface scattering in greater detail by considering different resistivity contributions of surface scattering for the two types of interfaces studied, i.e., $\mathrm{Cu} / \mathrm{SiO}_{2}$ and $\mathrm{Cu} / \mathrm{Ta}$. Further, we evaluate the impact of the roughnesses of the upper and lower $\mathrm{Cu}$ film surfaces on film resistivity. Because of these measures, this work is the most complete quantitative measurement of surface scattering in polycrystalline metals in which the resistivity contribution from grain-boundary scattering has been accurately determined. 


\section{RESISTIVITY MODELING}

We begin with a summary of the various physical models of the classical size effect to which the experimental results will be compared. Two important aspects of this summary are the examination of the temperature dependence of the resistivity for the various models, and the manner in which the various resistivity contributions are combined.

\section{A. Surface scattering in thin films}

The classical size effect was first modeled by Fuchs and Sondheimer (FS). ${ }^{5,6}$ The FS model is based on the Boltzmann transport theory and attributes resistivity increases in thin films and narrow lines to diffuse scattering of conduction electrons at the conductor's exterior surfaces with a probability of $1-p$, where $p$ is a specular scattering coefficient. An electron that scatters diffusely loses the additional momentum it has gained from the electric field and leaves the surface in a random direction. An electron that is specularly scattered does not change its momentum in the directions parallel to the surface, which include the direction of the electric field. Thus, specular scattering does not contribute to increased resistivity and the specular scattering coefficient is the model's most important parameter. It takes values between zero and one, and it is commonly varied to fit experimental data. The important length scales in this model are the conductor's dimension(s) perpendicular to the current flow (i.e., for a thin film, the thickness, $h$ ) and the mean free path of the conduction electrons due to phonon and impurity scattering, $\lambda$. The FS model describes the resistivity $\left(\rho_{\mathrm{FS}}\right)$ of a thin film as ${ }^{6}$

$\rho_{\mathrm{FS}}=\rho_{i}\left[1-\left(\frac{3}{2 k}\right)(1-p) \int_{1}^{\infty}\left(\frac{1}{t^{3}}-\frac{1}{t^{5}}\right) \frac{1-\exp (-k t)}{1-p \exp (-k t)} d t\right]^{-1}$,

where $k=h / \lambda$ and $\rho_{i}$ is the bulk resistivity of the metal. At room temperature, the bulk resistivity is primarily deter- mined by phonon scattering, while at low temperatures (4.2 $\mathrm{K})$, it is primarily determined by residual crystalline defects. In the limits of small $k$, Eq. (1a) is simplified into ${ }^{6}$

$$
\rho_{\mathrm{FS}}=\rho_{i}\left[1+\left(\frac{3}{8}\right) \frac{\lambda}{h}(1-p)\right] \text {. }
$$

It is convenient to consider the resistivity increase predicted by this model as $\Delta \rho_{\mathrm{FS}}$, which is given by $\Delta \rho_{\mathrm{FS}}=\rho_{\mathrm{FS}}-\rho_{i}$.

\section{B. Roughness induced surface scattering in thin films}

The FS model uses the specular reflection probability, $p$, as a variable fitting parameter, but does not explicitly include the roughness of the scattering surface. Soffer ${ }^{7}$ introduced a surface scattering model that uses the roughness of the scattering surface to calculate an angle-dependent specular reflection probability. This model allows for comparison to experimental data with no fitting parameters when the roughness of the scattering surfaces is known. For a thin film with the top surface (1) and the bottom surface (2) having separate root mean square roughness, $r_{1}$ and $r_{2}$, the Soffer specular reflection probability for each surface is given by ${ }^{8}$

$$
\begin{aligned}
& p_{1}(\cos \theta)=\exp \left[-\left(\frac{4 \pi r_{1}}{\lambda_{\mathrm{F}}}\right)^{2} \cos ^{2}(\theta)\right], \\
& p_{2}(\cos \theta)=\exp \left[-\left(\frac{4 \pi r_{2}}{\lambda_{\mathrm{F}}}\right)^{2} \cos ^{2}(\theta)\right],
\end{aligned}
$$

where $\theta$ is the angle of incidence of the electrons to the conductor's surface and $\lambda_{\mathrm{F}}$ is the electron wavelength at the Fermi surface, about $0.5 \mathrm{~nm}^{9}$ for $\mathrm{Cu}$. The average specularity parameter for the top and bottom surfaces in the Soffer model is given as

$$
\overline{p(\cos \theta)}=\frac{1}{2}\left[p_{1}(\cos \theta)+p_{2}(\cos \theta)\right]
$$

and Soffer's resistivity size effect model is then ${ }^{8}$

$$
\rho_{\text {Soffer }}=\rho_{i}\left\{\mathbf{1}-\left(\frac{3}{2 k}\right) \int_{0}^{1} \frac{\left(u-u^{3}\right)\left[1-\exp \left(-\frac{k}{u}\right)\right]\left\{1-\bar{p}(u)+\left[\bar{p}(u)-p_{1}(u) p_{2}(u)\right] \exp \left(-\frac{k}{u}\right)\right\}}{\left[1-p_{1}(u) p_{2}(u) \exp \left(-\frac{2 k}{u}\right)\right]} d u\right\}^{-1} .
$$

Using this model, the increase in resistivity due to surface roughness induced scattering is $\Delta \rho_{\text {Soffer }}=\rho_{\text {bulk }}$ $-\rho_{\text {Soffer. }}$

More recently, Rossnagel and $\mathrm{Kuan}^{10}$ (RK) proposed a semiempirical extension of the FS model to include surface roughness explicitly. Based on Monte Carlo simulations of electron trajectories near a rough surface, their resistivity model is given by

$$
\rho_{\mathrm{RK}}=\rho_{i}+\Delta \rho_{\mathrm{FS}}\left(1+\frac{r_{1}}{n_{\mathrm{RK}}}\right),
$$

where $n_{\mathrm{RK}}$ incorporates the conductor thickness and Fermi wavelength and is determined by Monte Carlo simulations of electron trajectories. This model continues to use the specularity coefficient of the FS model as a single fitting parameter and allows for larger resistivity increases than the FS model. 


\section{Grain-boundary scattering in thin films}

Mayadas and Shatzkes ${ }^{11}$ (MS) developed an extension of the Boltzmann transport theory to include reflection and transmission of conduction electrons at the grain boundaries of a polycrystalline metal. Their model assumes that grain boundaries are all either parallel or perpendicular to the direction of current flow and that electrons incident upon the parallel grain boundaries are only specularly reflected, i.e., the parallel grain boundaries have no role in the resistivity size effect. Each perpendicular grain boundary is treated as an internal surface, and when a conduction electron collides with the grain boundary, it has a probability of transmission or reflection that is quantified by a reflection coefficient, $R$. This coefficient is allowed to take values between zero and one and is commonly varied to fit experimental data. The important length scales for this model are the average grain size, $g$, and the electron mean free path, $\lambda$. The parameters $R$, $g$, and $\lambda$ are conveniently combined as $\alpha=(\lambda / g) R /(1-R)$ and the MS model describes the resistivity $\left(\rho_{\mathrm{MS}}\right)$ of a film as ${ }^{11}$

$$
\rho_{\mathrm{MS}}=\rho_{i}\left[1-\frac{3}{2} \alpha+3 \alpha^{2}-3 \alpha^{3} \ln \left(1+\frac{1}{\alpha}\right)\right]^{-1} .
$$

In the limits of small $\alpha$, Eq. (4a) is reduced to ${ }^{11}$

$$
\rho_{\mathrm{MS}}=\rho_{i}\left[1+\left(\frac{3}{2}\right)\left(\frac{\lambda}{g}\right)\left(\frac{R}{1-R}\right)\right] .
$$

Using the MS model, the increase in resistivity due to grain boundary scattering is $\Delta \rho_{\mathrm{MS}}=\rho_{\mathrm{MS}}-\rho_{i}$.

Equations (1b) and (4b) can be seen to have a fundamentally similar form, namely, $\rho(x)=\rho_{i}+A / x$, where $x$ is the experimentally varied size parameter (grain size or film thickness) and $A$ is a constant, typically determined by fitting to the experimental data. Given the tendency in polycrystalline thin films for the grain size and the film thickness to be nearly equal or at least proportional (i.e., $g \propto h$ ), the resistance increase associated with the classical size effect can be attributed to either mechanism when both effects are present. When the resistivity data comes from samples lacking independent variation in grain size and thickness, different combinations of $p$ and $R$ can provide the same value for the fitting constant, $A$, and thus fit the experimental data equally well. For example, Steinhögl et al. ${ }^{12}$ concluded that $p=0.6$ and $R=0.5$ best fitted their room temperature resistivity data of $230 \mathrm{~nm}$ high copper wires with widths ranging from 40 to $800 \mathrm{~nm}$, while assuming that the grain size equaled the smallest dimension of the wires. A later analysis by Marom and Eizenberg ${ }^{20}$ showed that specularity and reflection parameter pairs of $(p=0, R=0.42)$ and $(p=1, R=0.53)$ would fit Steinhögl et al.'s data as well.

\section{Matthiessen's rule and temperature dependence of the resistivity size effect}

An additional issue to consider is the applicability of Matthiessen's rule- the simple addition of the resistivities associated with different mechanisms. ${ }^{13}$ Impurity scattering and phonon scattering, as well as grain boundary and surface

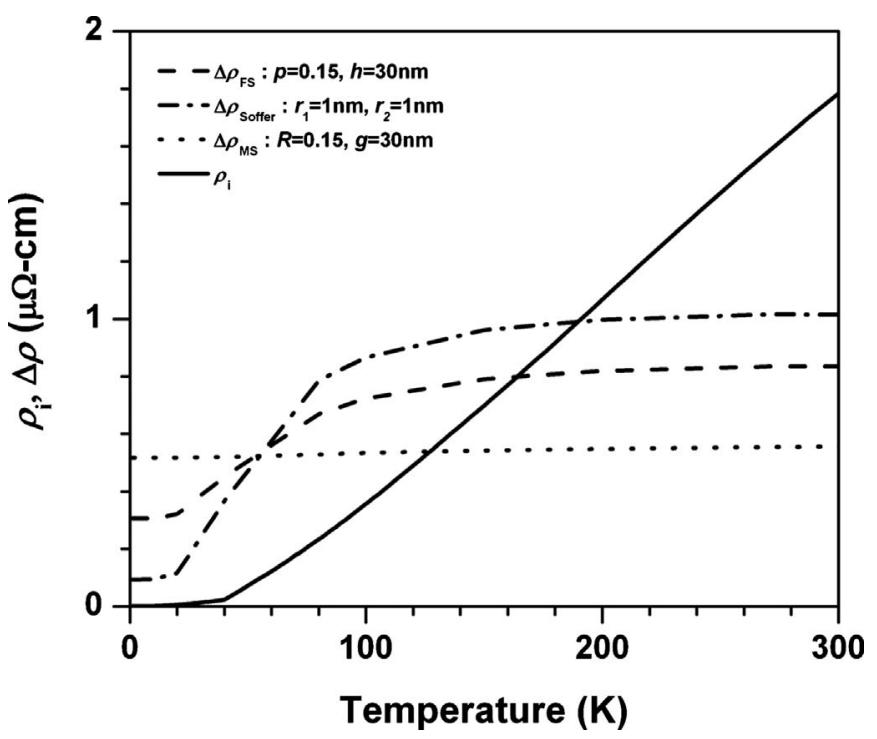

FIG. 1. A comparison of the temperature dependence of the resistivity increases predicted by the $\Delta \rho_{\mathrm{FS}}$ [using Eq. (1a)], $\Delta \rho_{\text {Soffer }}$ [using Eq. (2)], $\Delta \rho_{\mathrm{MS}}$ [using Eq. (4a)], and $\rho_{i}$ are plotted as a function of temperature for a hypothetical polycrystalline $\mathrm{Cu}$ thin film having a $30 \mathrm{~nm}$ grain size and a $30 \mathrm{~nm}$ thickness to illustrate their different temperature dependencies. The temperature dependence of the experimentally measured bulk resistivity, $\rho_{i}$, of $\mathrm{Cu}$ from Ref. 18 is also shown in the figure.

scattering, are often included in the modeling of resistivity size effects by the use of Matthiessen's rule. Landauer ${ }^{14}$ has pointed out that planar scattering defects (i.e., surfaces and grain boundaries) may not follow this rule, even though isotropic point scattering centers (impurities, phonons) do. Experimentally, significant deviations from Matthiessen's rule have been reported in studies of the classical resistivity size effect. ${ }^{15}$ In Fig. $1, \Delta \rho_{\mathrm{FS}}, \Delta \rho_{\mathrm{MS}}, \Delta \rho_{\mathrm{Soffer}}$, and $\rho_{i}$ are plotted as a function of temperature for a hypothetical film sample having a $30 \mathrm{~nm}$ grain size and a $30 \mathrm{~nm}$ thickness to illustrate the different temperature dependencies of the various models. As can be seen in the figure, $\Delta \rho_{\mathrm{MS}}$ increases $\sim 5 \%$ over the temperature range of $10 \mathrm{~K}$ to room temperature, and $\Delta \rho_{\mathrm{FS}}$ and $\Delta \rho_{\text {Soffer }}$ increase by more than $60 \%$, even though both grain size and thickness are held constant. A number of experimental works have attempted to separate the contributions of grain boundary scattering and surface scattering to the resistivity increase by comparing the experimental resistivity data over a range of temperatures to the different dependencies predicted by the models. ${ }^{16,17}$

The more significant dependency of $\Delta \rho_{\mathrm{FS}}$ and $\Delta \rho_{\mathrm{Soffer}}$ on temperature is due to the interaction between the surface scattering and phonon scattering (and to a lesser extent, grain boundary scattering and phonon scattering) that is inconsistent with Matthiessen's rule. Matthiessen's rule would require the simple addition of a constant surface or constant grain boundary resistivity contribution with the phonon contribution to provide the total resistivity. It should also be noted that the temperature dependence predicted for the FS $\left(\Delta \rho_{\mathrm{FS}}\right)$ and Soffer $\left(\Delta \rho_{\mathrm{Soffer}}\right)$ models differ significantly $(\sim 40 \%)$, even though a common physical mechanism is invoked. Surface scattering clearly shows a stronger interaction 
with phonon scattering (temperature dependence) than does grain boundary scattering and this is readily understood as the increased phonon scattering allows the fraction of electrons with momentum parallel to the external surfaces to be more frequently redirected toward the surfaces. For grain boundary scattering, the electrons cannot avoid impinging on grain boundaries, and, therefore phonon scattering, and thus temperature, have a small effect on the resistivity. In developing their model, Mayadas and Shatzkes assumed that the grain boundaries parallel to the current flow provided purely specular scattering of electrons. If these parallel boundaries were alternatively assumed to have a partially diffuse scattering character, they would provide a resistivity contribution that would be increased by phonon scattering and have similar temperature dependence as that of surface scattering. Such a resistivity contribution would scale with average grain size, rather than with sample thickness. This hypothesis is readily tested by consideration of a MS-type scattering model having different reflection coefficients at different temperatures. We will refer to this approach as the MST model when separate reflection coefficients for our RT and 4.2 K data are considered.

\section{E. Interactions between surface and grain boundary scattering}

As discussed before, Matthiessen's rule is not valid for combining surface and phonon scattering. The next question is whether surface and grain boundary scattering would also violate Matthiessen's rule. An interaction between surface and grain boundary scattering may be expected at low temperatures, where grain boundary scattering might serve instead of phonon scattering to allow the electrons with momentum parallel to the external surfaces to be more frequently redirected toward the surfaces. In addition to providing their model for grain boundary and phonon scattering, Mayadas and Shatzkes ${ }^{11}$ derived a more complex model that combines the scattering effects of grain boundaries, external surfaces, and phonons in polycrystalline metallic films incorporating the interactions between these mechanisms. The total film resistivity of the Mayadas Shatzkes Surface model (MSS) is computed as ${ }^{11}$

$$
\begin{aligned}
\rho_{\mathrm{MSS}}= & {\left[\frac{1}{\rho_{\mathrm{MS}}}-\left(\frac{6}{\pi k \rho_{i}}\right)(1-p) \int_{0}^{\pi / 2} d \phi \int_{1}^{\infty} d t \frac{\cos ^{2} \phi}{\mathrm{H}^{2}(t, \phi)}\right.} \\
& \left.\times\left(\frac{1}{t^{3}}-\frac{1}{t^{5}}\right) \frac{1-\exp [-k t \mathrm{H}(t, \phi)]}{1-p \exp -k t \mathrm{H}(t, \phi)]} d t\right]
\end{aligned}
$$

where

$$
\mathrm{H}(t, \phi)=1+\frac{\alpha}{\cos \phi \sqrt{1-1 / t^{2}}} .
$$

For a single crystal film $(\alpha=0)$, and Eq. (5) reduces to Eq. (1a). For polycrystalline thin films, Eq. (5) includes the resistivity increase due to grain boundary scattering redirecting some of the electrons toward the surfaces.

The more commonly used approach for considering combined surface and grain boundary scattering mechanisms is to assume that surface and grain boundary mechanisms are

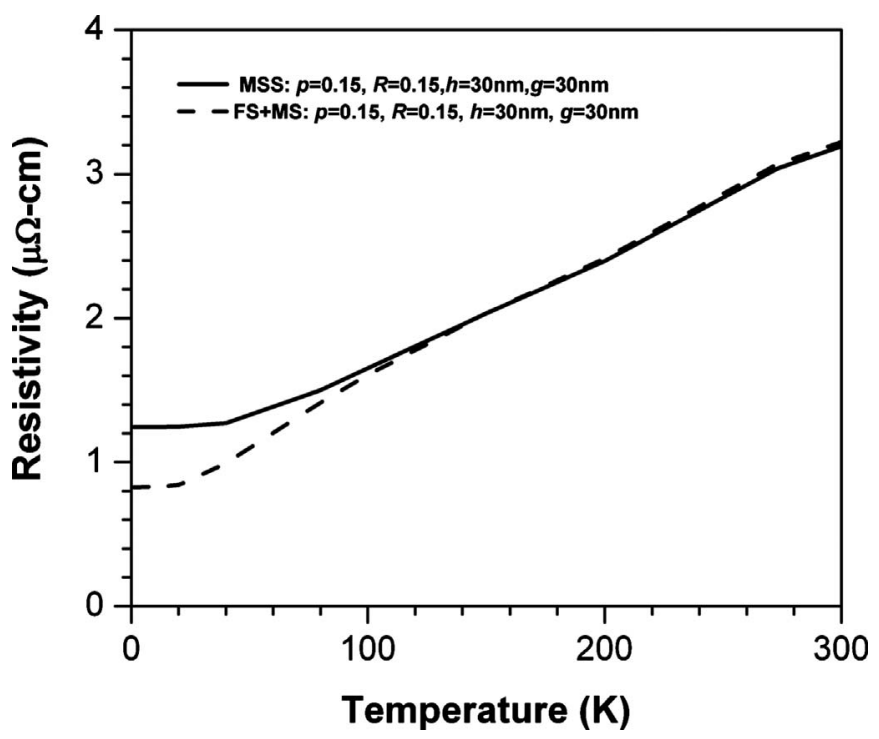

FIG. 2. Temperature dependence of the total resistivity of two combined surface and grain boundary scattering models for a $\mathrm{Cu}$ thin film with a thickness, $h$, and a grain size, $g$, of $30 \mathrm{~nm}$. The MSS model refers to the model described in Eq. (5) in which higher order interactions between the two additional scattering mechanisms are considered. The FS + MS model refers to the simple Matthiessen's rule combination [Eq. (6a)] of these two scattering effects. For both models, the surface specularity parameter, $p$, and the grain boundary reflection coefficient, $R$, are set equal to 0.15 .

independent, and so Matthiessen's rule can apply. A combined model (FS+MS) for the FS surface, grain boundary, and phonon scattering using this approach can be written as

$$
\rho_{\mathrm{FS}+\mathrm{MS}}=\rho_{i}+\Delta \rho_{\mathrm{FS}}+\Delta \rho_{\mathrm{MS}}
$$

This equation includes the interaction between phonon scattering and surface scattering and the interaction between phonon scattering and grain boundary scattering but neglects the interaction between grain boundary and surface scattering. In a similar fashion, a combined model (Soffer+MS) for roughness induced surface, grain boundary, and phonon scattering using Matthiessen's rule can be written as

$$
\rho_{\text {Soffer+MS }}=\rho_{i}+\Delta \rho_{\text {Soffer }}+\Delta \rho_{\mathrm{MS}},
$$

and similarly for a combined RK roughness and grain boundary scattering,

$$
\rho_{\mathrm{RK}+\mathrm{MS}}=\rho_{\mathrm{RK}}+\Delta \rho_{\mathrm{MS}} .
$$

Figure 2 is a comparison between the combined FS and MS models using Matthiessen's rule [Eq. (6)] and the MSS model described by Eq. (5) for the hypothetical case of a 30 -nm-thick film with $30 \mathrm{~nm}$ grain size. The interaction between grain boundary and surface scattering is evident in the higher low-temperature resistivity $(0.4 \mu \Omega \mathrm{cm}$, about $40 \%$, at $10 \mathrm{~K}$ ) of the MSS model, wherein the scattering of electrons with momentum parallel to the external surfaces by grain boundaries results in additional surface scattering. This higher order interaction is absent in the FS+MS model. At higher temperatures, the scattering of the electrons with momentum parallel to the surfaces is primarily due to phonons 
in both models and thus negligible resistivity differences are predicted.

Another complication in comparisons between experimental data and the various models is the choice of an appropriate value for the bulk resistivity of $\mathrm{Cu}, \rho_{i}$. Ideally, sufficiently high purity $\mathrm{Cu}$ samples are used, where impurity scattering is negligible, and the bulk room temperature $(293 \mathrm{~K})$ value due to phonon scattering can be used, $\rho_{i}=1.67 \mu \Omega \mathrm{cm}$. However, this value is rarely used and $\rho_{i}$, is more often considered an additional free fitting parameter. For example, Marom and Eizenberg used $\rho_{i}=2.3 \mu \Omega \mathrm{cm}$ to fit resistivities of copper wires with widths between 100 to $200 \mathrm{~nm}$ and heights of 150 to $300 \mathrm{~nm}$. They then concluded that surface scattering is fully diffuse $(p=0.0)$. In the work presented here, the experimentally measured ${ }^{18}$ resistivity of bulk $\mathrm{Cu}, \rho_{i}$, as a function of temperature is used throughout to calculate the temperature dependent mean free path (arising from phonon scattering) for the conduction electrons using the relationship: $\rho_{i} \lambda_{=} 6.6 \times 10^{-14} \Omega \mathrm{m}^{2}{ }^{11,16}$ A plot of the resistivity of bulk $\mathrm{Cu}$ as a function of temperature is included in Fig. 1.

\section{F. Scattering from impurities and voids}

The presence of impurities and voids can increase the resistance of polycrystalline conductors over and above that from surface and grain boundary scattering. Higher concentrations of impurities, such as $\mathrm{Al}, \mathrm{O}$, and $\mathrm{C}$, have been observed in thin films and a modest resistivity increase of $\sim 0.01 \mu \Omega \mathrm{cm}$ has been attributed to impurity scattering in high purity $(\geq 99.99 \%) \mathrm{Cu}$ thin films. ${ }^{19}$ Other studies of the classical size effect have reported very substantial contributions to resistivity from impurity scattering, but only rarely have the sample impurities been separately measured. ${ }^{20,21}$ Voids are also often found in thin films as a by-product of processing, and while typically not of the length scale to affect resistivity directly, voids can result in substantial errors in the measurement of sheet resistance that is used to experimentally calculate resistivity. Characterization of sample void volumes is, unfortunately, also rarely included in experimental reports. While extreme voiding (void area fraction $>50 \%$ ) can often be observed by visual inspection, intermediate levels of voiding that are not evident without electron microscopies can be a significant part of the resistance increase observed. An example of this is Hensel's experimental data ${ }^{22}$ of an apparently increased resistivity for thinner epitaxial $\mathrm{CoSi}_{2}$ films, which he attributed to a reduced quality (pinhole voids) of these films and not to a resistivity size effect. Extreme voiding results in a catastrophic increase in film resistance as the film morphology approaches a percolation limit and this regime has been modeled by several workers. ${ }^{23,24}$ However, the resistivity error expected from low levels of voiding has not been previously considered and a brief description of a simple model of this case is provided in the Appendix.

\section{EXPERIMENTS}

$\mathrm{SiO}_{2}$ encapsulated $\mathrm{Cu}$ thin films, with and without $\mathrm{Ta}$ barriers, were prepared on $\mathrm{Si}$ (100) substrates having a 150-nm-thick layer of thermally grown $\mathrm{SiO}_{2}$. Prior to film deposition, the substrates were rf sputter-cleaned and cooled to $-40{ }^{\circ} \mathrm{C}$ by contact with a liquid nitrogen cooled $\mathrm{Cu}$ plate. An underlayer of $20 \mathrm{~nm}$ of $\mathrm{SiO}_{2}$ was rf sputter deposited prior to the $\mathrm{Cu}$ film deposition and a $20 \mathrm{~nm} \mathrm{SiO}_{2}$ overlayer was subsequently sputter deposited to form a $\mathrm{SiO}_{2} / \mathrm{Cu} / \mathrm{SiO}_{2}$ structure. The $\mathrm{Cu}$ layers were deposited by dc sputter deposition from high purity $(99.9999 \%) \mathrm{Cu}$ targets and had thicknesses in the range of 28 to $158 \mathrm{~nm}$. For films having the $\mathrm{SiO}_{2} / \mathrm{Ta} / \mathrm{Cu} / \mathrm{Ta} / \mathrm{SiO}_{2}$ structure, a $2 \mathrm{~nm}$ Ta layer was dc sputter deposited immediately prior to, and again after, the $\mathrm{Cu}$ layer deposition. These structures were used to meet the requirement of having identical scattering interfaces for the top and bottom surfaces of the film. Samples were subjected to various annealing temperatures under a reducing gas environment of $\mathrm{Ar}+3 \% \mathrm{H}_{2}$ to obtain different grain sizes at each thickness to meet the requirement of having independent grain size and film thickness variation in the sample set. It should be noted that the encapsulation also served to increase the extent of grain growth that could be induced by annealing without significant void formation. ${ }^{25}$ Samples were annealed at $150{ }^{\circ} \mathrm{C}$ and $600{ }^{\circ} \mathrm{C}$ for $30 \mathrm{~min}$ in a tube furnace, and at $400{ }^{\circ} \mathrm{C}$ for $6 \mathrm{~s}$ by halogen lamp heating (rapid thermal annealing). Table I contains a summary of samples fabricated, along with their respective annealing temperature, thickness, root mean square roughness of the top $\left(r_{1}\right)$ and bottom $\left(r_{2}\right) \mathrm{Cu}$ /encapsulant interfaces, resistivity, and average grain size. The sheet resistance of the samples at 4.2 K was measured by dipping a Van der Pauw geometry four point probe ${ }^{26}$ into liquid helium while using a Keithley 2400 Source meter and 2182 nanovoltmeter for data collection. As will be described below, the low-temperature resistivity of the $\mathrm{Cu}$ films was consistent with high purity films, i.e., no significant resistivity contribution from impurity scattering was observed. Compositional profiling with secondary ion mass spectrometry was also used to examine $\mathrm{Cu}$ film purity. The major impurity observed in the $\mathrm{Cu}$ layer was oxygen, which was found to have an upper bound of $30 \mathrm{ppm}$, but a lower limit could not be established because of the presence of residual oxygen from the encapsulation layer.

The experimental requirement for thorough structural characterization of the sample film was also met. Details of preparation and examination of electron transparent samples for microstructural characterization are given elsewhere. ${ }^{4}$ For the measurement of void fraction, the samples were examined by high angle annular dark field (HAADF) imaging in scanning transmission electron microscopy (TEM) mode at relatively low magnifications. This void area quantification technique was also used to guide the development of the deposition and processing techniques described above. The void fractions were found to be between 0 and $2.4 \%$. The resistivity errors that these void area fractions can give rise to are described in the Appendix and correspond to negligibly small error (i.e., a $0.4 \%$ resistance error for the case of a $2.4 \%$ void area fraction).

For grain size measurement, the samples were examined by hollow cone dark field (HCDF) imaging in TEM mode to provide the highest diffraction contrast, ${ }^{25}$ as shown in Fig. 3. By imaging the given field of view at three different sample 
TABLE I. Annealing temperature, thickness, root mean square roughnesses (upper, $\mathrm{r}_{1}$, and lower, $\mathrm{r}_{2}$, of the $\mathrm{Cu}$ /encapsulant layer interfaces), 296 and $4.2 \mathrm{~K}$ resistivity, and grain size data for: $\mathrm{SiO}_{2}$-encapsulated $\mathrm{Cu}$ thin films (a), and the $\mathrm{Ta} / \mathrm{SiO}_{2}$-encapsulated $\mathrm{Cu}$ thin films (b) (Refs. 3, 4, and 35).

\begin{tabular}{|c|c|c|c|c|c|c|c|}
\hline $\begin{array}{c}\text { Anneal } \\
\left({ }^{\circ} \mathrm{C}\right)\end{array}$ & $\begin{array}{c}\text { Thickness } \\
(\mathrm{nm})\end{array}$ & $\begin{array}{c}\mathrm{r}_{1} \\
(\mathrm{~nm})\end{array}$ & $\begin{array}{c}\mathrm{r}_{2} \\
(\mathrm{~nm})\end{array}$ & $\begin{array}{c}\rho \text { at } 296 \mathrm{~K} \\
(\mu \Omega \mathrm{cm})\end{array}$ & $\begin{array}{c}\rho \text { at } 4.2 \mathrm{~K} \\
(\mu \Omega \mathrm{cm})\end{array}$ & $\begin{array}{l}\text { Grain dia. } \\
\quad(\mathrm{nm})\end{array}$ & Grains measured \\
\hline \multicolumn{8}{|c|}{ (a) $\mathrm{SiO}_{2} / \mathrm{Cu} / \mathrm{SiO}_{2}$} \\
\hline 150 & 27.0 & 1.2 & 0.8 & 3.99 & 2.04 & $40.5 \pm 2.5$ & 483 \\
\hline 150 & 31.6 & 1.4 & 0.8 & 3.63 & 1.62 & $47.7 \pm 2.8$ & 525 \\
\hline 150 & 35.3 & 1.1 & 1.0 & 3.20 & 1.30 & $54.3 \pm 2.1$ & 1363 \\
\hline 150 & 37.1 & 0.9 & 0.8 & 3.08 & 1.14 & $64.8 \pm 2.5$ & 1362 \\
\hline 150 & 45.1 & 1.0 & 0.8 & 2.75 & 0.90 & $101.1 \pm 4.6$ & 919 \\
\hline 150 & 71.8 & 0.6 & 1.5 & 2.30 & 0.52 & $171.7 \pm 7.9$ & 872 \\
\hline 150 & 136.7 & 1.2 & 2.0 & 2.06 & 0.27 & $342.2 \pm 20.1$ & 525 \\
\hline 150 & 143.9 & 0.9 & 1.3 & 2.01 & 0.25 & $248.0 \pm 17.2$ & 412 \\
\hline 400 & 41.7 & 1.0 & 0.7 & 3.05 & 0.95 & $87.7 \pm 3.2$ & 1563 \\
\hline 400 & 83.6 & 0.6 & 1.1 & 2.25 & 0.36 & $221.5 \pm 10.7$ & 785 \\
\hline 400 & 157.9 & 0.5 & 2.0 & 1.92 & 0.19 & $419.3 \pm 21.8$ & 662 \\
\hline 600 & 33.6 & 0.2 & 0.7 & 2.94 & 0.92 & $68.4 \pm 4.4$ & 452 \\
\hline 600 & 36.9 & 0.5 & 1.0 & 2.70 & 0.78 & $81.4 \pm 4.5$ & 576 \\
\hline 600 & 46.4 & 0.4 & 0.9 & 2.54 & 0.58 & $112.6 \pm 7.7$ & 419 \\
\hline 600 & 74.5 & 0.3 & 1.0 & 2.25 & 0.34 & $220.0 \pm 9.5$ & 1045 \\
\hline 600 & 149.7 & 0.3 & 1.2 & 1.94 & 0.16 & $425.2 \pm 15.7$ & 1518 \\
\hline \multicolumn{8}{|c|}{ (b) $\mathrm{SiO}_{2} / \mathrm{Ta} / \mathrm{Cu} / \mathrm{Ta} / \mathrm{SiO}_{2}$} \\
\hline 600 & 28.3 & 0.8 & 1.1 & 4.08 & 1.82 & $34.6 \pm 1.5$ & 960 \\
\hline 600 & 34.2 & 1.1 & 1.2 & 3.73 & 1.76 & $39.4 \pm 1.7$ & 1020 \\
\hline 600 & 38.7 & 1.3 & 1.3 & 3.69 & 1.68 & $44.3 \pm 2.2$ & 743 \\
\hline 600 & 48.4 & 1.0 & 1.0 & 2.95 & 0.99 & $69.6 \pm 3.4$ & 776 \\
\hline 600 & 77.9 & 1.4 & 1.2 & 2.55 & 0.68 & $110.1 \pm 4.6$ & 1129 \\
\hline 600 & 153.1 & 0.9 & 1.5 & 2.08 & 0.32 & $345.1 \pm 15$ & 1033 \\
\hline
\end{tabular}

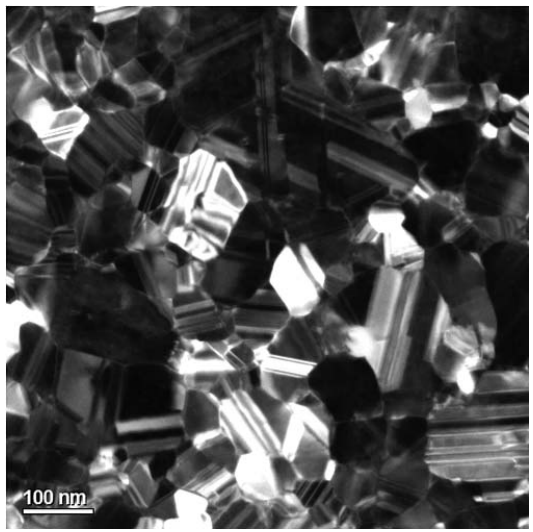

(a) -2 degree tilt

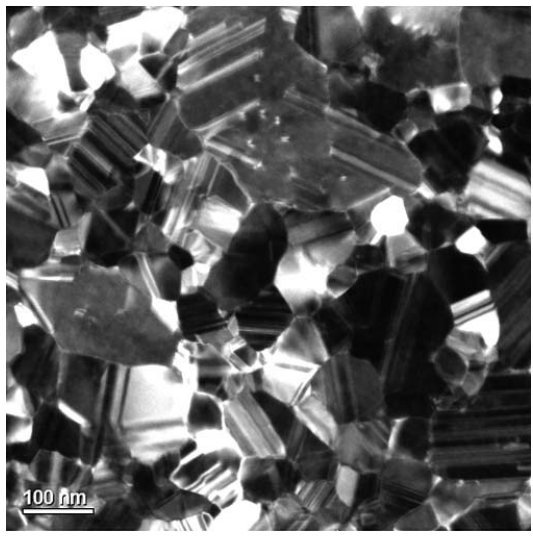

(b) 0 degree tilt

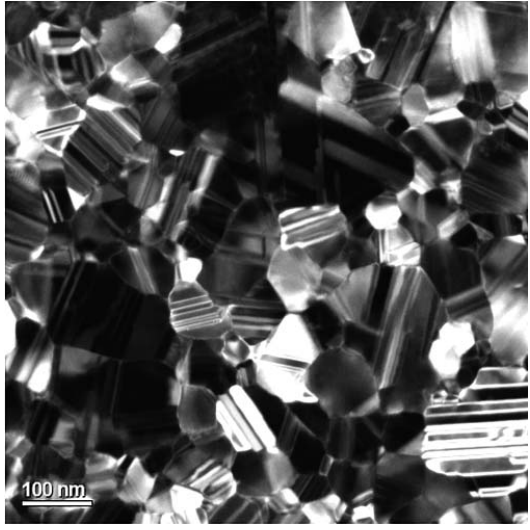

(c) 2 degree tilt

FIG. 3. Hollow cone dark field (HCDF) transmission electron micrographs of a 33.6-nm-thick $\mathrm{Cu}$ film encapsulated in $20 \mathrm{~nm} \mathrm{SiO}_{2}$ and annealed at $600{ }^{\circ} \mathrm{C}$, imaged at three different sample tilts of the same field of view. The three tilts allow the grain boundaries to be identified for hand tracing. The hand-traced boundary network is used for automated measurement of grain size. 

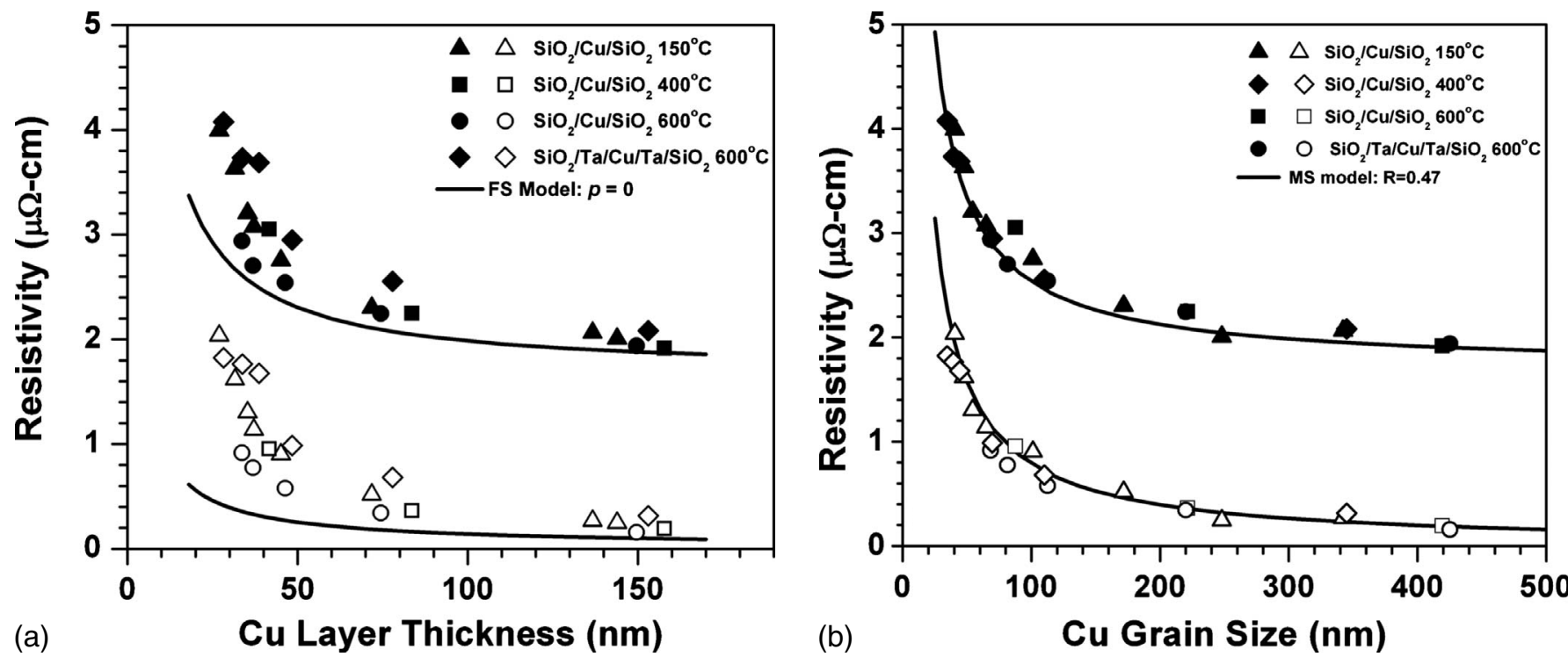

FIG. 4. The total resistivity of $\mathrm{SiO}_{2} / \mathrm{Cu} / \mathrm{SiO}_{2}$ and $\mathrm{SiO}_{2} / \mathrm{Ta} / \mathrm{Cu} / \mathrm{Ta} / \mathrm{SiO}_{2}$ thin films (a) as a function of $\mathrm{Cu}$ layer thickness, $h$, and (b) as a function of $\mathrm{Cu}$ layer grain size, $g$. The filled symbols are resistivities measured at room temperature, and the open symbols are resistivities measured at $4.2 \mathrm{~K}$. The data points correspond to the samples listed in Table I. The solid curve corresponds to the FS model [Eq. (1a)] in (a) and MS [Eq. (4a)] model in (b).

tilts (Fig. 3) and thereby varying the diffraction contrast, it was possible to visually identify the grains. Twin boundaries within grains were excluded in the traced boundary network, since this type of grain boundary has been reported to have a minimal contribution to sample resistivity. ${ }^{27}$ The reported average grain size for each sample is the diameter of the equivalent circle with area equal to the average of the grain areas. The errors on this mean are quoted as $2 \sigma$ values at a $95 \%$ confidence level for the given grain population. ${ }^{28}$

To quantify the roughnesses of the buried $\mathrm{Cu} / \mathrm{SiO}_{2}$ and $\mathrm{Cu} / \mathrm{Ta}$ interfaces $\mathrm{x}$-ray reflectivity experiments were performed at the Stanford Synchrotron Radiation Lightsource (SSRL). The $\mathrm{Cu}$ film thickness and the roughness of the upper and lower $\mathrm{Cu}$ /encapsulant layer interfaces were determined by fitting of the reflectivity data to established models..$^{29,30}$ The reflectivity data were collected on the thin film diffraction beam line $2-1$. This beam line is equipped with a Huber 2-circle goniometer, a pair of $1 \mathrm{~mm}$ slits as the analyzer, ${ }^{31}$ and a He filled sample stage which was used to decrease the air scattering background. The $1.549 \AA$ wavelength $\mathrm{x}$-rays were monochromated with double bounce $\mathrm{Si}(111)$ crystals. Two types of scans were performed: specular, where $\omega=2 \theta / 2=\theta$, and off-specular where $\omega=2 \theta / 2 \pm 0.15^{\circ}$. The off-specular scans were used to subtract the contribution of diffusely scattered $\mathrm{x}$-rays to the specular reflection resulting in a purely specular reflectivity pattern. Data were collected from $2 \theta=0.2^{\circ}$ to $12^{\circ}$, with a step size of $0.02^{\circ}, 0.01^{\circ}$, or $0.005^{\circ}$ depending on film thickness.

\section{RESULTS AND DISCUSSION}

In order to separate the effect of grain boundary scattering from that of surface scattering, the grain size of the $\mathrm{Cu}$ samples should ideally be varied independent of film thick- ness, an example of which was shown in our previous work. ${ }^{3,4}$ As can be seen in Table I, we have produced a wide range of grain sizes for films at each thickness (from $\sim 1.2$ $\times$ thickness to $\sim 3.1 \times$ thickness), although a fully independent variation was not achieved. Nevertheless, with the careful quantification of grain size exercised in this work (error less than $\sim 7 \%$ ), this degree of independent variation is sufficient for the separate quantification of grain boundary and surface scattering effects.

In Fig. 4(a), the resistivities of the $\mathrm{SiO}_{2} / \mathrm{Cu} / \mathrm{SiO}_{2}$ and $\mathrm{SiO}_{2} / \mathrm{Ta} / \mathrm{Cu} / \mathrm{Ta} / \mathrm{SiO}_{2}$ samples are plotted as a function of thickness at both room temperature (RT), typically $296 \mathrm{~K}$ in our laboratory, and at 4.2 K. The FS model [using Eq. (1a)] is shown by the lines in the figure and fails to describe the experiment even with the most extreme value for the specularity coefficient $(p=0)$. Within the $\mathrm{SiO}_{2} / \mathrm{Cu} / \mathrm{SiO}_{2}$ samples, it can be seen that for samples of similar thickness the resistivity decreases with increasing annealing temperature. Further, the $\mathrm{Cu}$ samples with Ta interlayers are found to have consistently higher resistivities than those without Ta. The presence of samples with different resistivities at each thickness indicates that surface scattering alone cannot explain the resistivity size effect in these $\mathrm{Cu}$ thin films.

Figure 4(b) is a plot of the room and low temperature resistivity as a function of grain size. It is immediately evident from the figure that the additional variations in resistivity associated with the annealing temperature or the presence of Ta are no longer present, but are instead accounted for by sample grain size. Figure 4(b) compares both the room temperature and low temperature experimental data with the predictions of the MS model of Eq. (4a) with a reflection coefficient of $R=0.47$ and no other variable parameters. The MS model fits the data well. However, it can be observed that experimental resistivities at room temperature tend to be generally higher than the MS model prediction while the 
TABLE II. For a series of classical size effect models, the model fitting parameters with their optimized values, the number of fitting parameters, a, the sum of the residual squared errors (SSE) and the calculated value of the Bayesian information criterion (BIC) are given. The BIC is calculated using Eq. (7). The models examined are the Soffer Model [Eq. (2)], the FS model [Eq. (1a)], the MS model [Eq. (4a)], the Soffer + MS combined model [Eq. (6b)], MSS combined model [Eq. (5)], the MST model [Eq. (4a) with separate reflection coefficients at room temperature and $4.2 \mathrm{~K}]$, the FS + MS combined model using Matthiessen's rule [Eq. (6a)], the FS+MS combined model [Eq. (6a) but with different specularity coefficients for $\mathrm{Cu} / \mathrm{SiO}_{2}$ and $\mathrm{Cu} / \mathrm{Ta} / \mathrm{SiO}_{2}$ caps], and the $\mathrm{RK}+\mathrm{MS}$ model [Eq. (6c)]. See the text for more detail.

\begin{tabular}{lccrr}
\hline \hline Model name & Model parameters & Number of parameters, $a$ & $\begin{array}{c}\text { SSE } \\
\left(\mu \Omega^{2} \mathrm{~cm}^{2}\right)\end{array}$ & BIC \\
\hline Soffer & none & 0 & 30.5 & 108.8 \\
FS & $p=0$ & 1 & 22.9 & 99.8 \\
MS & $R=0.47$ & 1 & 0.96 & -39.4 \\
Soffer+MS & $R=0.38$ & 1 & 2.39 & 0.4 \\
MSS & $p=0.61 R=0.42$ & 2 & 0.85 & -41.4 \\
MST & $R_{R T}=0.49 R_{4.2 K}=0.45$ & 2 & 0.59 & -57.4 \\
FS+MS & $p=0.52 R=0.43$ & 2 & 0.48 & -66.1 \\
FS+MS (Caps $)$ & $p_{\mathrm{SiO}_{2}}=0.51 p_{\mathrm{Ta}}=0.55 R=0.43$ & 3 & 0.48 & -62.2 \\
RK+MS & $p=0.68 R=0.43 n_{\mathrm{RK}}=18.2$ & 3 & 0.43 & -67.5 \\
\hline \hline
\end{tabular}

resistivities at $4.2 \mathrm{~K}$ are lower, i.e., the temperature dependence of the resistivity is not well described. This may reflect limitations inherent in the MS model (e.g., assumption of purely specular scattering from parallel grain boundaries or the use of a temperature independent reflection coefficient) or this may indicate the presence of a surface scattering contribution to the resistivity, such as that given by the FS model, which has significant temperature dependence (Fig. 1).

To assess the limits of our experimental data in understanding the resistivity size effect, the data at both temperatures were compared to the models described in Sec. II. The models were: the Soffer model [Eq. (2)], the FS model [Eq. (1a)], the MS model [Eq. (4a)], the Soffer+MS model [Eq. (6b)], the MSS model [Eq. (5)], the MST model [Eq. (4a)] with separate reflection coefficients at room temperature and $4.2 \mathrm{~K}$ ], the FS+MS model [Eq. (6a)], the FS + MS model with "Caps" [Eq. (6a) but with different specularity coefficients for $\mathrm{Cu} / \mathrm{SiO}_{2}$ and $\mathrm{Cu} / \mathrm{Ta} / \mathrm{SiO}_{2}$ interfaces] and the RK+MS model [Eq. (6c)].

In the fitting of FS+MS models, two different assumptions regarding the surface scattering were considered. In one case, identical scattering from the $\mathrm{Cu} / \mathrm{SiO}_{2}$ and the $\mathrm{Cu} / \mathrm{Ta}$ interfaces was assumed and a single specularity parameter was used for the combined data. In the second case, two separate specularity parameters were used for the $\mathrm{Cu} / \mathrm{SiO}_{2}$ and for the $\mathrm{Cu} / \mathrm{Ta}$ interfaces and this model variation is identified as "Caps."

The model parameters were obtained by minimizing the sum squared error (SSE). At RT, a fixed bulk resistivity value of $1.7 \mu \Omega \mathrm{cm}$ and an electron mean free path of 39 $\mathrm{nm}$ were used for all models. At $4.2 \mathrm{~K}$, a fixed resistivity of $0.002 \mu \Omega \mathrm{cm}$ and an electron mean free path of $33 \mu \mathrm{m}$ were used. ${ }^{18}$ As noted in Sec. II E, these $\rho_{i}$ values were those experimentally determined from high purity bulk $\mathrm{Cu}^{18}$ and were not a varied fitting parameter. The minimum SSE for each of these resistivity models is listed in Table II along with the optimum values of the model parameters used to obtain this global minimum within the physical limits allowed by each model, e.g., $0<p<1$. Since the SSE can be reduced and the goodness-of-fit improved by introducing additional fitting parameters, we use the Bayesian information criterion (BIC) (Refs. 32-34) for model comparison and selection. The BIC incorporates a penalty term for an increased number of fitting parameters, and, thus, is a suitable criterion for comparing models with different numbers of adjustable parameters. The formulation of the BIC used, assuming the errors to be normally distributed, is:

$$
\begin{aligned}
B I C & =-2 \times \ln (L)+a \ln (n) \\
& =n \ln \left(\frac{\mathrm{SSE}}{n}\right)+n \ln (2 \pi)+n+a \ln (n),
\end{aligned}
$$

where $L$ is the overall likelihood (i.e., the product of the likelihoods for each of the measurements), $a$ is the number of adjustable or fitting parameters, and $n$ is the number of experimental measurements $(n=44,22$ measurements at each of two temperatures), and

$$
\frac{\mathrm{SSE}}{n}=\sigma^{2}=\frac{\sum_{i=1}^{n}\left(\rho_{i}^{\text {experiment }}-\rho_{i}^{\text {model }}\right)^{2}}{n} .
$$

For this formulation of the BIC, good models have negative BIC's, and, the lower the BIC, the better the model. The difference between the BIC values of two models must be greater than two for one model to be considered a better predictor of the experimental behavior than another. When the magnitude of the difference between the values of the BIC for two models is less than two, it is not possible to distinguish between the two models. The BIC values for the nine models that were considered are given in Table II. 
The residual error from fitting experimental data to any physical model is not only a result of experimental error, but it is also the result of the limitations of the physical model. The large summed squared error of $22.9 \mu \Omega^{2} \mathrm{~cm}^{2}$ and the large positive BIC of 99.8 for the FS model indicate that it clearly fails to describe the experiment, even with the most extreme value allowed for the specularity coefficient $(p=0)$. This conclusion could also be drawn from inspection of Fig. 4(a). The Soffer model for surface scattering also fails to describe the data. It has a larger summed squared error of $30.5 \mu \Omega^{2} \mathrm{~cm}^{2}$ and a large BIC of 108.8. As is also seen in Fig. 4, the MS grain boundary scattering model [Fig. 4(b)] is a dramatic improvement over the FS surface scattering model [Fig. 4(a)]. Table II gives a BIC of -39.4 for the MS model, which indicates that it is a good model for the observed resistivity behavior. However, a considerable SSE of $0.96 \mu \Omega^{2} \mathrm{~cm}^{2}$ remains due, in part, to the inability of the MS model to account for the experimental drop in $\Delta \rho$ with temperature.

The MST model (an SSE of $0.59 \mu \Omega^{2} \mathrm{~cm}^{2}$ and BIC of -57.4) allows the temperature dependence of the resistivity data to be better fitted by using separate (thus temperature dependent) grain boundary reflection coefficients at $4.2 \mathrm{~K}$ and at RT. This does provide a significantly better model for the data than the MS model, and establishes that the MS model alone does not provide the correct temperature dependence. However, there are other physical models that give the correct temperature dependence. The FS+MS model (using a simple Matthiessen's rule addition of surface and grain boundary scattering effects) with a SSE of $0.48 \mu \Omega^{2} \mathrm{~cm}^{2}$ and a BIC of -66.1 provides a significantly improved fit over both the MS and MST models. Based on this improved fit, we conclude that thickness dependence is present in the experimental data. Given this thickness dependence, there is no need to invoke additional temperature dependence to the MS model with the MST model. The presence of both temperature dependence and residual thickness dependence in our experimental data confirms the presence of a weak surface scattering contribution (over and above that from grain boundary scattering) to the size effect in $\mathrm{Cu}$.

Table II gives the grain boundary scattering reflection coefficient of 0.43 and surface scattering specularity coefficient of 0.52 for the combined FS+MS model. This intermediate value for the specularity coefficient is in contrast to the many previous works, wherein a surface specularity coefficient of zero was assumed, which simply maximized the surface scattering contribution to the resistivity increase. ${ }^{20,16}$ For our samples, the partition into surface and grain boundary contributions to the resistivity size effect provides averages of $27 \%$ from surface scattering and $73 \%$ from grain boundary scattering for room temperature. At $4.2 \mathrm{~K}$, the contribution from surface scattering is $14 \%$, while that from grain boundary scattering is $86 \%$.

To further explore the role of the interface properties on surface scattering, the FS+MS model was extended to include two specularity coefficients for the two interfaces we used $\left(p_{\mathrm{SiO}_{2}}\right.$ and $p_{\mathrm{Ta}}$, for $\mathrm{Cu} / \mathrm{SiO}_{2}$ and $\mathrm{Cu} / \mathrm{Ta}$ interfaces, respectively). Surprisingly, this did not improve the SSE, nor did it change the grain boundary reflection coefficient from $0.43 \mu \Omega^{2} \mathrm{~cm}^{2}$. It did, however, degrade the BIC to -62.2 from -66.1 for the FS+MS model (due to more fitting parameters). The lack of improvement in the SSE and the increase in the BIC confirm that, in spite of the higher resistivities observed for the $\mathrm{SiO}_{2} / \mathrm{Ta} / \mathrm{Cu} / \mathrm{Ta} / \mathrm{SiO}_{2}$ samples, little of the resistivity increase can be attributed to differences in the surface scattering of conduction electrons at the $\mathrm{Cu} / \mathrm{Ta}$ and $\mathrm{Cu} / \mathrm{SiO}_{2}$ interfaces. Rather, the resistivity increase is a simple consequence of the smaller grain size present in the $\mathrm{SiO}_{2} / \mathrm{Ta} / \mathrm{Cu} / \mathrm{Ta} / \mathrm{SiO}_{2}$ samples (Table I).

The role of interfacial roughness was further explored by comparing our data to the Soffer + MS and RK+MS models. The Soffer+MS model is a poorer description of the observed resistivity behavior (SSE of $2.39 \mu \Omega^{2} \mathrm{~cm}^{2}$, BIC of 0.4$)$ than the MS model by itself $\left(0.96 \mu \Omega^{2} \mathrm{~cm}^{2}\right.$, BIC -39.4) due, in part, to its temperature dependence. From this, we conclude that the Soffer model fails to correctly describe the physics of the resistivity size effect. However, the RK + MS model does reduce the summed squared residual error from that of the FS+MS $\left(0.48 \mu \Omega^{2} \mathrm{~cm}^{2}\right)$ to $0.43 \mu \Omega^{2} \mathrm{~cm}^{2}$. This fitting was performed with the thickness parameter, $n_{\mathrm{RK}}$, of $18.2 \mathrm{~nm}$, where $n_{\mathrm{RK}}$ was determined by minimization of the residual error instead of by a Monte Carlo simulation. While this reduction in error justifies additional efforts to relate surface roughness to resistivity, it is worth noting that the improvement observed was not statistically significant. This point is underscored by the fact that the BIC of -67.5 for the (3 parameter) $\mathrm{RK}+\mathrm{MS}$ model is not significantly lower than the BIC of -66.1 for the (2 parameter) $\mathrm{FS}+\mathrm{MS}$ model (i.e., the magnitude of the difference in the BIC's is less than two).

The MSS model differs from the FS + MS model in that it includes the interaction between grain boundary and surface scattering. The optimum parameters for the MSS model to describe our data are $p=0.61$ and $R=0.42$. However, with a SSE of $0.85 \mu \Omega^{2} \mathrm{~cm}^{2}$, the MSS model is a significantly worse description of our data than the FS+MS model, primarily as it overestimates resistivities at low temperature (as shown in Fig. 2). Additionally, the BIC of -41.4 for the MSS model is significantly worse than that for the FS+MS model. Thus, we conclude that the interaction of surface and grain boundary scattering postulated by Mayadas and Shatzkes and expected to be evident at low temperatures (in the absence of phonon scattering) does not occur.

The microstructure of our samples is columnar: the grains extend from the top to the bottom external surfaces and all of the grain boundaries are primarily perpendicular to the external surfaces. The observed absence of increased surface scattering due to the presence of grain boundaries (a lack of interaction) indicates that while the grain boundary scattering in our samples necessarily changes the in-film-plane component of electron momentum, it does not significantly increase the out-of-film-plane component, normal to the external surfaces. This means that electrons that are in initial states having momentum parallel to the external surfaces are scattered into final states having momentum parallel to the external surfaces. In other words, if the electron was not traveling toward the surface when it encountered the grain boundary, it will not be sent toward the surface after scattering from the grain boundary. By contrast, Mayadas and Shatzkes assumed that the electrons that scattered at the grain boundaries did so 
into all possible momentum states, similar to a "diffuse" surface scattering event. While we do not hypothesize that grain boundary scattering is "specular," it does appear that the components of electron momentum that are parallel to the grain boundary planes are largely conserved. Note that this is different from the case of a diffuse scattering event at the conductor's external surface which does not preserve the momentum components parallel to the surface. This lack of the expected interactions explains the observed simple summation of surface and grain boundary resistivities and suggests a fundamental difference between surface and grain boundary scattering.

For applications of nanoscale conductors, these results are significant. The quantification of grain boundary and surface scattering provides a basis for accurate estimation of the resistivity increases to be expected as conductor dimensions are further reduced with semiconductor device scaling. Surface scattering has been found to be largely independent of the chemistry of the external surface $\left(\mathrm{Cu} / \mathrm{SiO}_{2}\right.$ and $\mathrm{Cu} / \mathrm{Ta}$ providing similar results), but it is possibly sensitive to the topography of the surface. The lack of interaction between grain boundary scattering and surface scattering suggests that these interactions may also be absent for narrow lines, where the scattering from surfaces that define the sides of the line (sidewalls) gives rise to an additional complication. If the sidewall scattering of lines is assumed to be similar to that of the top and bottom surfaces of our films, and is an additive resistivity effect (no interaction), then grain boundary scattering is expected to be the dominant resistivity size effect for lines having equal height, width, and grain size. This is not unexpected, as all conduction electrons must cross grain boundaries to contribute to the current in the line. This suggests that practical efforts to reduce the resistivity of narrow lines should be directed toward processing changes to increase the conductor grain size rather than efforts to change the conductor external surface and interface chemistries. Areas for further study include the effect of surface roughness, and geometrical studies to quantify the extent of interaction between sidewalls and top and bottom external surfaces.

\section{SUMMARY AND CONCLUSIONS}

In summary, we have carefully studied the contributions of surface scattering and grain boundary scattering to the resistivity increase observed with reduction in conductor thickness in polycrystalline $\mathrm{Cu}$ thin films through quantitative measurement of the primary experimental variables and comparison of the data to a number of accepted models. The samples studied were relatively large grained, having an average grain size greater than the film thickness. We extended our previous result that grain boundary scattering was the dominant scattering mechanism and provided an unambiguous experimental verification of the grain boundary scattering model of Mayadas and Shatzkes. ${ }^{11}$ We were additionally able to provide a measurement of the surface scattering for our samples and found it to be well described by the FuchsSondheimer model with an intermediate value, $p=0.52$, for the surface specularity coefficient. This value is in contrast to prior reports where fully diffuse scattering surfaces (coeffi- cient of zero) were claimed. We also found that the increase in surface scattering at low temperatures modeled by Mayadas and Shatzkes and resulting from grain boundary scattering was not evident. The surface scattering from $\mathrm{Cu} / \mathrm{SiO}_{2}$ and $\mathrm{Cu} / \mathrm{Ta}$ interfaces was found to be indistinguishable. Our data does suggest roughness dependence to the surface scattering, but this was not conclusively demonstrated. Voids and impurities were found to have negligible impact on the measured resistivities.

\section{ACKNOWLEDGMENTS}

We thank H. Seltman for his guidance on the statistical analysis, A. D. Darbal, V. Kumar, and S. Roberts for their assistance with the grain size data, D. C. Berry for rapid thermal annealing, C. Fredricksen for his assistance with the low temperature measurements, and our colleagues for many fruitful discussions. We gratefully acknowledge the financial support of the Semiconductor Research Corporation, Task 1292.008, and partial support from the MRSEC program of the NSF under Grant No. DMR-0520425. Portions of this research were carried out at the Stanford Synchrotron Radiation Lightsource (SSRL), a national user facility operated by Stanford University on behalf of the U.S. Department of Energy, Office of Basic Energy Sciences.

\section{APPENDIX}

While resistivity models for highly voided (near percolation threshold) thin films can be found in the literature, ${ }^{23,24}$ there is no published model suitable to describe the case of a low density of isolated voids, as we observe. This may be due to the relative simplicity of the calculation, which is presented below to show the small contribution of the voids to the resistivity increase observed in our work.

We can consider the resistance of a thin film of resistivity $\rho$, to consist of many identical area elements, each of length, $L$, width, $W$, and thickness, $h$. The resistance, $R_{0}$, of each element is then just

$$
R_{0}=\rho \frac{L}{W \times h},
$$

where we have assumed the direction of current flow to be parallel to the dimension $L$. We can model the formation of voids in a film consisting of many such elements by adding a square hole (of equal width and length, $\delta$, ) in one corner of each area element. This geometry is shown in Fig. 5 for clarity. The additional scattering from the walls of the void are neglected, since this is a small fraction of the total conductor surface area $(\delta \ll L, W)$. The voided area fraction is

$$
\% \text { Voided Area }=\frac{\delta^{2}}{L \times W} \times 100
$$

The resistance of the area element with the void can be calculated by considering the element to consist of two resistances in series (see Fig. 5). The first resistance is the region indicated by " 3 " in the figure, which has a resistance, $R_{3}$, parallel to the $L$ dimension, given by 


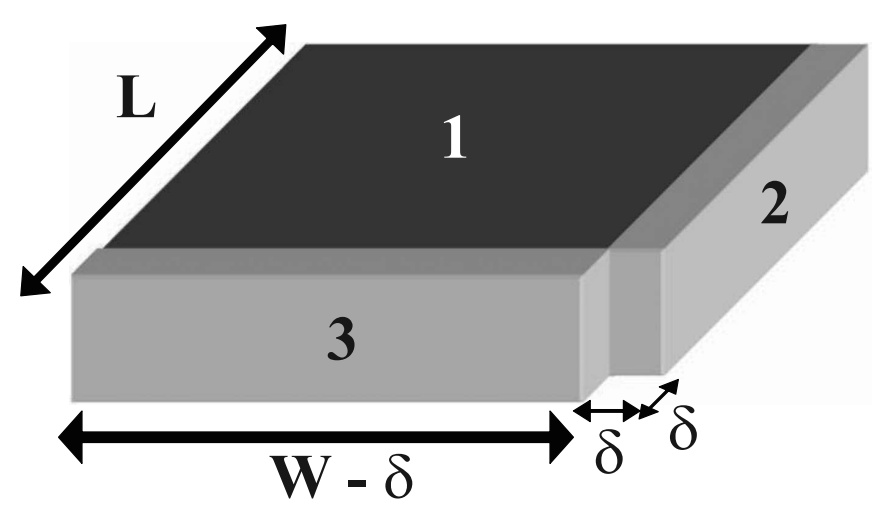

FIG. 5. Geometry of resistivity model for small void area fractions.

$$
R_{3}=\rho \frac{\delta}{(W-\delta) \times h_{R}} .
$$

Please note that in this equation $h_{R}$ is the increased thickness of all three regions resulting from the redistribution of the $\mathrm{Cu}$ atoms. We will require the total volume of each area element to remain constant, independent of void area, to conserve the amount of $\mathrm{Cu}$ present in the film, and we will use this condition later to calculate $h_{R}$.

The second resistance in series consists of regions 1 and 2 combined, $R_{1,2}$, and this has a resistance parallel to the $L$ dimension of

$$
R_{1,2}=\rho \frac{(L-\delta)}{W \times h_{R}} .
$$

The relative increase in resistance of each area element (and hence of the film) is given by

$$
\text { Resistance Fraction Increase }=\frac{R_{1,2}+R_{3}-R_{0}}{R_{0}},
$$

from which $h_{R}$ is determined by conserving the volume of our area element. The volume of the unvoided area element is just $L \times W \times h$ and the volume of the area element with a void is given by $\left[(L \times W)-\delta^{2}\right] \times h_{R}$. Equating these gives

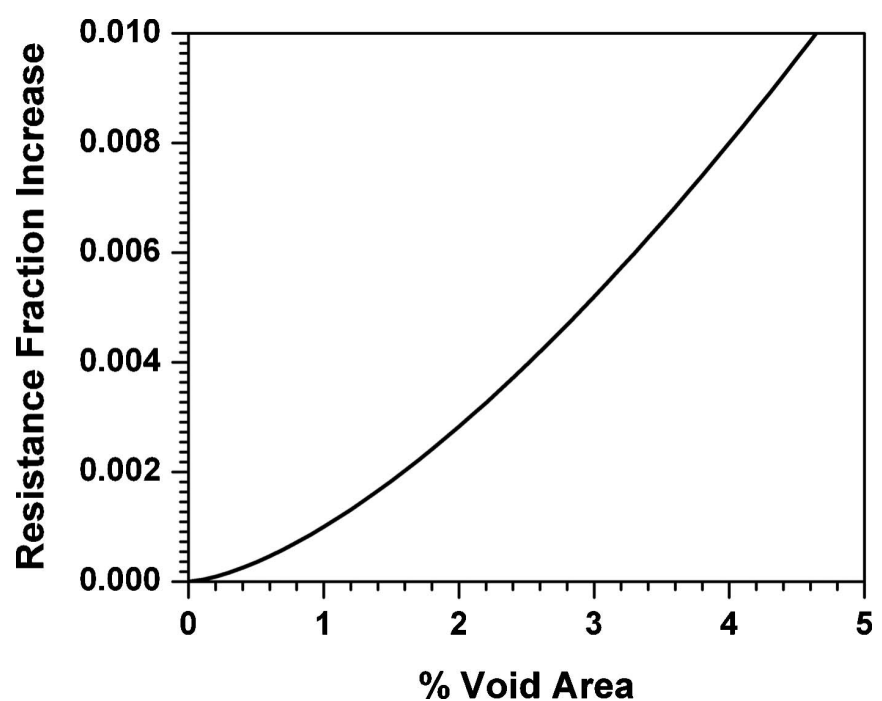

FIG. 6. Fractional increase in resistance as a function of voided area percentage.

$$
\frac{h_{R}}{h}=\frac{L \times W}{(L \times W)-\delta^{2}} .
$$

A plot of fraction of Resistance Increase as a function of $\%$ Voided Area calculated using the above equations is shown in Fig. 6.

These equations can be simplified further by taking $L=W$ and by setting $\frac{\delta}{L}=x$. After algebraic manipulation, this gives a very simple result,

$$
\text { Resistance Fraction Increase }=x^{3} \text {. }
$$

As the Fraction Voided Area $=x^{2}$, this gives the simple geometrical result:

Fraction Resistivity Increase $=(\text { Fraction Voided Area })^{1.5}$.

This model is clearly limited to a low density of isolated (noninteracting) voids, and requires $\delta \ll L$ in order for the current crowding near the edges of a void area to be neglected. For films with low void fractions (less than 5\%), the resistivity error is less than $1 \%$ and negligible.
${ }^{1}$ J. J. Thomson, Proc. Cambridge Philos. Soc. 11, 120 (1901).

${ }^{2}$ J. R. Sambles, Thin Solid Films 106, 321 (1983).

${ }^{3}$ T. Sun, B. Yao, A. P. Warren, K. Barmak, M. F. Toney, R. E. Peale, and K. R. Coffey, Phys. Rev. B 79, 041402 (2009).

${ }^{4}$ T. Sun, B. Yao, A. P. Warren, V. Kumar, S. Roberts, K. Barmak, and K. R. Coffey, J. Vac. Sci. Technol. A 26, 605 (2008).

${ }^{5}$ K. Fuchs, Proc. Cambridge Philos. Soc. 34, 100 (1938).

${ }^{6}$ E. H. Sondheimer, Adv. Phys. 1, 1 (1952).

${ }^{7}$ S. G. Soffer, J. Appl. Phys. 38, 1710 (1967).

${ }^{8}$ J. R. Sambles and K. C. Elsom, J. Phys. D 15, 1459 (1982).

${ }^{9}$ H. Marom and M. Eizenberg, J. Appl. Phys. 99, 123705 (2006).

${ }^{10}$ S. M. Rossnagel and T. S. Kuan, J. Vac. Sci. Technol. B 22, 240 (2004).
${ }^{11}$ A. F. Mayadas and M. Shatzkes, Phys. Rev. B 1, 1382 (1970).

${ }^{12}$ W. Steinhögl, G. Schindler, G. Steinlesberger, and M. Engelhardt, Phys. Rev. B 66, 075414 (2002).

${ }^{13}$ N. W. Ashcroft and N. D. Mermin, Solid State Physics (Saunders College, Philadelphia, 1976), pp. 323-324.

${ }^{14}$ R. Landauer, IBM J. Res. Dev. 1, 223 (1957).

${ }^{15}$ R. C. Munoz, C. Arenas, G. Kremer, and L. Moraga, J. Phys.: Condens. Matter 15, L177 (2003).

${ }^{16}$ W. Zhang, S. H. Brongersma, Z. Li, D. Li, O. Richard, and K. Maex, J. Appl. Phys. 101, 063703 (2007).

${ }^{17}$ P. M. Th. M. Van Attekum, P. H. Woerlee, G. C. Verkade, and A. A. M. Hoeben, Phys. Rev. B 29, 645 (1984).

${ }^{18}$ D. R. Lide, CRC Handbook of Chemistry and Physics, 87 th ed. 
(CRC-Press, Boca Raton, 2006), pp. 12-39.

${ }^{19}$ J.-W. Lim and M. Isshiki, J. Appl. Phys. 99, 094909 (2006).

${ }^{20}$ H. Marom, J. Mullin, and M. Eizenberg, Phys. Rev. B 74, 045411 (2006).

${ }^{21}$ J. J. Plombon, E. Andideh, V. M. Dubin, and J. Maiz, Appl. Phys. Lett. 89, 113124 (2006).

${ }^{22}$ J. C. Hensel, R. T. Tung, J. M. Poate, and F. C. Unterwald, Phys. Rev. Lett. 54, 1840 (1985).

${ }^{23}$ C. Pennetta, L. Reggiani, and Gy. Trefán, Phys. Rev. Lett. 84, 5006 (2000).

${ }^{24}$ K. Sieradzki, K. Bailey, and T. L. Alford, Appl. Phys. Lett. 79, 3401 (2001).

${ }^{25}$ B. Yao, T. Sun, V. Kumar, K. Barmak, and K. R. Coffey, J. Mater. Res. 23, 2033 (2008).

${ }^{26}$ L. J. Van der Pauw, Philips Res. Rep. 13, 1 (1958).

${ }^{27}$ L. Lu, Y. Shen, X. Chen, L. Qian, and K. Lu, Science 304, 422 (2004).

${ }^{28}$ D. T. Carpenter, J. M. Rickman, and K. Barmak, J. Appl. Phys. 84, 5843 (1998).
${ }^{29}$ M. Wormington, Ch. Panaccione, K. M. Matney, and D. K. Bowen, Philos. Trans. R. Soc. London, Ser. A 357, 2827 (1999).

${ }^{30}$ S. K. Sinha, E. B. Sirota, S. Garoff, and H. B. Stanley, Phys. Rev. B 38, 2297 (1988).

${ }^{31} \mathrm{http}: / / \mathrm{www}-\mathrm{ssrl} . \mathrm{slac}$. stanford.edu/beamlines/bl2-1/

${ }^{32}$ G. E. Schwarz, Ann. Stat. 6, 461 (1978).

${ }^{33}$ A. E. Raftery, Biometrika 83, 251 (1996).

${ }^{34}$ C. T. Volinsky and A. E. Raftery, Biometrics 56, 256 (2000).

${ }^{35}$ The values shown in Table I differ in detail from similar tables in references 3 and 21 due to the inclusion of additional grain size and x-ray reflectivity data. These changes resulted in minimal quantitative changes to the optimized model parameters calculated. For example, Ref. 21 reports a MS reflection coefficient of $0.50 \pm 0.03$ to describe room temperature resistivity data and this may be compared to the room temperature MST model value of 0.49 given in the current work. Similarly, Ref. 3 reports a low temperature MS reflection coefficient of 0.44 and this can be compared to the low temperature MST model value of 0.43 given in the current work. 